\title{
The Training of Computational Thinking in Basic Computer Teaching in Universities
}

\author{
Jian Peng \\ Jiangxi Vocational Technical College of Industry \& Trade, Nanchang, Jiangxi,330038 \\ 3107000933@qq.com
}

\begin{abstract}
In the present stage of basic university computer teaching, training students' computational thinking has become the most important one, on the one hand, it is because training students' computational thinking can strengthen students' thinking logic ability, also can improve students' ability to solve problems, on the other hand, it is also to strengthen students' comprehensive computer ability.
\end{abstract}

Keywords: Computer basic teaching, computational thinking, training

\section{大学计算机基础教学中的计算思维培养}

\author{
彭健
}

江西工业贸易职业技术学院 南昌 江西 330038

3107000933@qq.com

\section{摘要}

在现阶段的大学计算机基础教学中，培养学生的计算思维成为了重中之重，一方面是因为培养学生的 计算思维能够增强学生的思维逻辑能力, 也能够提高学生解决问题的能力, 另一方面也是为了增强学 生的综合计算机能力, 而本文就主要围绕大学计算机基础教学中对学生的计算思维的培养进行探讨。

关键词: 计算机基础教学; 计算思维; 培养

\section{1. 计算机的思维的基本概述和计算机思维培 养的现状}

\section{1 计算思维的基本概述}

计算思维是一种思维活动, 而计算思维中也包含计 算思维能力和计算思维意识以及计算思维方法。而计算 思维意识所指的是人类的无意识科学思维活动。而通过 计算机基础教学, 培养学生的计算思维, 其实指的就是 激发学生的科学思维活动, 让学生将这种科学思维运用 于现实中, 并解决现实问题。而计算思维方法其实指的 就是计算机科学自身的方法。想要通过计算机基础教学 培养学生的计算思维, 首先就应该拓展学生的思维能 力, 不局限学生的思维发展, 让学生能够尽可能的发散 自己的思维, 并独立解决问题。教师应该给予学生一定 的指导, 但是教师不应该干涉学生解决问题的过程, 而 是应该尽可能地引导学生, 如果教师总是代替学生解决 问题, 那么学生的思维能力和解决问题的能力就无法得 到锻炼, 而且解决问题的能力从某种方面来说, 也是学 生的实践能力。

\section{2 大学计算机基础教学中计算思维培养的 现状分析}

有关于对学生计算思维的培养, 在计算机技术教学 中, 难以取得理想的成绩, 而会导致这种情况出现的原 因也有很多种。例如高校忽略了对学生的计算思维的培 养, 过于看重学生的理论成绩。却没有意识到计算思维 对于计算机人才而言的重要性。即使是对于非计算机专 业的学生而言, 计算思维也有其一定的科学性。而作为 人类三大思维, 计算思维, 理论思维和实验思维, 理论 思维明显是目前最受人们关注的存在, 但是计算思维和 实验思维也不容忽视。高校应该加强对计算思维的认 识, 并加强对学生计算思维的培养。除此之外, 高校还 应该加强对学生理论知识基础的提升。学生如果无法掌 握好计算机理论基础, 那么也难以提升计算机技术应用 能力，而教育部门目前也已经颁发了一系列文件，用以 加强高校对学生计算思维的培养的重视。 


\begin{tabular}{|c|c|c|c|}
\hline \multicolumn{4}{|c|}{ 大学计算机基础教学计算思维培养的策略分析 } \\
\hline $\begin{array}{l}\text { 重视计算 } \\
\text { 思维意识 } \\
\text { 的培养 }\end{array}$ & $\begin{array}{l}\text { 计算思维 } \\
\text { 与专业实 } \\
\text { 践的结合 }\end{array}$ & $\begin{array}{l}\text { 注重教学 } \\
\text { 中实验教 } \\
\text { 学的方式 }\end{array}$ & $\begin{array}{l}\text { 考核评价方 } \\
\text { 式的完善 }\end{array}$ \\
\hline $\begin{array}{l}\text { 针对计算 } \\
\text { 思维培养 } \\
\text { 的现状, } \\
\text { 首先要求 } \\
\text { 院校及教 } \\
\text { 师充分重 } \\
\text { 视计算思 } \\
\text { 维的重要 } \\
\text { 性, 可在 } \\
\text { 计算机基 } \\
\text { 础教学内 } \\
\text { 容中进行 } \\
\text { 知识内容 } \\
\text { 的划分, } \\
\text { 将其中所 } \\
\text { 缊含的思 } \\
\text { 维有意识 } \\
\text { 的深入教 } \\
\text { 学中。 }\end{array}$ & $\begin{array}{l}\text { 需正确把 } \\
\text { 握计算思 } \\
\text { 维与专业 } \\
\text { 实践的结 } \\
\text { 合, 如关 } \\
\text { 于网络内 } \\
\text { 容教学过 } \\
\text { 程中, 可 } \\
\text { 利用 SOC } \\
\text { 的方式使 } \\
\text { 其重点体 } \\
\text { 现在应用 } \\
\text { 知识或网 } \\
\text { 络组成等 } \\
\text { 方面, 并 } \\
\text { 将常见的 } \\
\text { 网络连接 } \\
\text { 与实际应 } \\
\text { 用方式作 } \\
\text { 出分析。 }\end{array}$ & $\begin{array}{c}\text { 首先需将 } \\
\text { 计算维 } \\
\text { 引入基础 } \\
\text { 规范实验 } \\
\text { 过程中, 以 } \\
\text { 此发挥计 } \\
\text { 算思维的 } \\
\text { 引导作用。 } \\
\text { 其次利用 } \\
\text { 构造性的 } \\
\text { 实验方式 } \\
\text { 帮助学生 } \\
\text { 能够运用 } \\
\text { 计算思维 } \\
\text { 方式处理 } \\
\text { 问题。 }\end{array}$ & $\begin{array}{c}\text { 为使学生综 } \\
\text { 合能力进一 } \\
\text { 步提升, 可 } \\
\text { 对考试内容 } \\
\text { 进一步丰 } \\
\text { 富, 如对管 } \\
\text { 理类专业学 } \\
\text { 生考试过程 } \\
\text { 中, 可要求 } \\
\text { 学生利用计 } \\
\text { 算机基础知 } \\
\text { 识与管理专 } \\
\text { 业知识进行 } \\
\text { 企业的设计 } \\
\text { 与管理思 } \\
\text { 想。 }\end{array}$ \\
\hline
\end{tabular}

\begin{tabular}{|c|c|}
\hline \multicolumn{2}{|c|}{$\begin{array}{c}\text { 计算机的思维的基本概述和计算机思维培养的现 } \\
\text { 状 }\end{array}$} \\
\hline 计算思维的基本概述 & $\begin{array}{l}\text { 大学计算机基础教学中 } \\
\text { 计算思维培养的现状分 } \\
\text { 析 }\end{array}$ \\
\hline 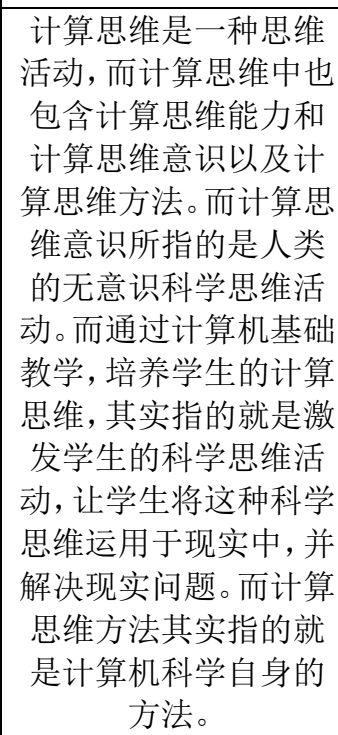 & $\begin{array}{c}\text { 有关于对学生计算思维的 } \\
\text { 培养, 在计算机技术教学 } \\
\text { 中, 难以取得理想的成绩, } \\
\text { 而会导致这种情况出现的 } \\
\text { 原因也有很多种。例如高 } \\
\text { 校忽略了对学生的计算思 } \\
\text { 维的培养, 过于看重学生 } \\
\text { 的理论成绩。却没有意识 } \\
\text { 到计算思维对于计算机人 } \\
\text { 才而言的重要性。 }\end{array}$ \\
\hline
\end{tabular}

\section{2 计算机的思维的基本概述和计算机思维培 养的现状}

\section{1 重视计算思维意识的培养}

培养学生的计算思维, 必须要通过计算机技术课程 教学平台, 因为有关对学生计算思维的培养并不会单独 成课, 而主要是通过学生对计算机基础知识的学习来挖 掘学生的计算思维, 并提升学生的计算思维, 当然, 因 为目前而言, 计算思维培养还没有得到高校和教师的重 视, 所以计算思维培养的效果也不是非常显著, 但是只 要教师和高校意识到了计算思维的重要性, 并将对学生 计算思维的培养和计算机基础教学内容结合在一起, 就 能够有效提升学生的计算思维, 并引导学生将理论转化 为实践。

\section{2 计算思维与专业实践的结合}

基于计算机基础教学之上发展的对学生计算思维 的培养, 主要针对的是非计算机专业的学生, 而如果教 师在进行计算机基础教学时, 只一昧地向学生灌输理论 知识, 那么不一定能够调动学生对计算机学习的兴趣, 还会影响到对学生计算思维的培养, 因为计算思维是应 用思维, 而纯粹的理论教学是无法将应用思维的作用体 现出来的, 因此教师在进行计算机基础教学时, 也应该 结合实践, 让学生能够应用计算思维, 解决现实问题。

\section{3 注重教学中实验教学的方式}

在大学计算机基础教学中实验教学的重要性是母 庸置疑的, 通过实验教学能够培养学生的计算思维, 也 能够提高学生的实践能力, 而在学生进行实践的过程 中, 学生的计算思维又能够得到进一步的深化, 而且, 计算思维也能够有利于学生解决问题。所以在教师对学 生进行实验教学时, 教师就应该引导学生投入到实验教 学中, 并激发学生的计算思维, 让学生用计算思维解决 问题。除此之外, 实验教学也能够提升学生的创新意识, 而将创新意识和计算思维结合在一起, 能够使学生的计 算机应用能力得到更进一步的拓展。而在实验教学中所 设置的实验应该具备探索性, 让学生能够对此感兴趣, 并让学生在不断探索中掌握更多的计算机内容。

\section{4 考核评价方式的完善}

就目前而言, 大部分高校所采取的计算机技术教学 的考核方式都是机考和卷面考试相结合, 而在机考和卷 面考试中就能够考察学生的实践能力和理论知识掌握 程度, 但是这样的考试方式, 也是十分片面的, 并不能 完全体现出学生的综合计算机能力, 因此高校还应该丰 
富计算机基础教学的考试内容, 最好结合学生的专业, 让学生能够加深对自己本专业的了解和掌握, 也让学生 能够加强计算机应用能力。而同时学校还应该扩大对学 生的计算机能力考核的范围, 不仅仅应该对学生进行最 终的成绩评定, 也应该对学生进行日常表现的考核, 所 谓的日常表现考核也就是指对学生的课堂表现情况和 学生的作业完成情况的考核, 通过这两个方面的考核也 能够体现出学生的学习态度和学生的学习效率。

\section{3. 计算思维与教学方案研究}

随着高科技的发展, 我国高等教育在教学过程中会 随着时代进步的发展, 而不断的改变教学方式方法。而 对于改革的教学方法并不是指在教学过程中单纯的以 传统的教学方法以及学习模式, 而是将新的方式方法融 入到以前的教学方法当中, 并且在教师教学方法的过程 当中又能够融入新的教学方式方法, 以追随时代的发 展。我国高等教育教学对于计算机教学的主体主要是以 计算机思维的方式来引导学生对计算思维以及计算机 方法的基础掌握。帮助学生能够更好的进行计算思维学 习。

\section{1 教学内容设计的基础性}

我国对于计算思维的发展，从古时候就已经开始， 因为计算思维与人们的生活、学习、工作等都有密切的 关系, 计算思维充斥着人们的整个生活, 它也是人们生 活当中必不可缺的一种生活技能。因此我国高等教育在 对大学生进行计算思维教学的过程当中, 更注重稳固对 大学生计算机的基础教学。因此, 我国大部分高等学校 在进行计算思维教学过程当中主要是以大学计算机基 础教学为主体, 通过对传统的计算机思维教学方法进行 创新。在教学的过程中, 主要是引导学生对计算思维的 基础性学习, 帮助大学生能够更好地理解掌握计算思 维。

\section{2 教学内容设计的启发性}

计算机基础课程在我国高校当中通常会被设为公 共课, 并且计算机基础课程相对于其它教学课程而言也 是具有一定的特别性。由于学生对于课程学习的出发点 不同, 以及对于课程理解的想法不同。因此, 如果高校 只是单纯的依赖于计算机基础课本对大学生进行教学, 并不能达到一种特别好的教学效果, 尤其是对于计算机 理论部分教学, 大部分大学生普遍对于计算机理论学习 会感到枯燥、乏味。也因此会给大学生学习计算机基础 课程造成一定的影响。所以高校教师在进行大学计算机 基础课程教学过程中要有效的设计正确的教学方法, 并 通过一些趣味性的教学方式, 激发大学生对于计算思维 的思考, 进而提高学生对于大学计算机基础课程的学习 兴趣。

\section{3 教学内容设计的综合性和使用性}

课堂教学的性质对于大学生的人生发展具有重要 的影响。然而在我国高校课堂教学过程当中, 大部分高 校教室在课堂教学过程当中普遍都过于枯燥, 只是一味 的给学生灌输课本知识, 没有很好的激发大学生对于学 习的兴趣。因此, 教师如何在规定时间内的课堂教学当 中, 启发大学生的学习思维显得尤为重要。教师在计算 机基础课程教学过程当中需要占据主导地位, 要引导学 生如何去学习。由于计算机思维相对比较抽象, 但是计 算思维与当代人们生活紧密联系, 因此, 教师在对大学 生进行计算机基础课程教学的过程当中, 要通过运用生 活当中的实际例子对大学生进行教学, 以帮助大学生能 够更好的理解教学课程内容, 以及掌握教学课程知识。 当然, 由于社会的快速发展对于人才也提出了更高的要 求, 所以教师在对大学生进行计算机基础教学过程当 中, 要注重于对大学生进行综合性的教学, 以复合型人 才为主体, 对大学生进行人才培养。

\section{4. 教学评价体系}

计算机基础课程相对于其他课程来说具有较强的 操作性, 因此, 教师在对计算机专业的学生进行评价过 程当中, 不仅仅要对这类学生进行计算机理论的评价, 还要更注重对大学生对计算机操作性的评价。然而因为 计算机技术课程具有较强的操作性, 所以更能检验大学 生对于计算机基础课程的学习效果, 以及大学生对于 计算思维的掌握能力。

对于大学生而言, 随着社会高科技的发展, 大学生 对于计算机技术能力的掌握也越来越重要, 因此高校对 于大学计算机基础课程的开设目的就是为了培养大学 生计算思维能力。然而学生在进行计算机基础课程的学 习过程当中, 也要积极主动的学习, 计算机基础课程的 知识内容。只有这样才能发挥高校对于计算机技术课程 开设的真正意义。

\section{5. 基于大学生计算思维培养的课程训练}

我国高校大部分学生对于计算思维的掌握力度现 象不太乐观，并且随着社会时代的发展，对于计算机的 掌握能力, 已经成为了人们生活当中必不可缺的一部 分, 因此, 我国以及高校对于计算机思维教学的改革也 越来越重视, 所以高校在进行计算机基础教学过程当 中, 以计算机基础教学课程为主体, 引导大学生对于计 算机思维的学习。提高大学生对于计算机思维的理解和 认识, 加强大学生对于计算机操作的能力。

\section{1 具有针对性的教学组织过程}

高校对于计算机技术课程的开设的主要目标就是 为了能够培养大学生的计算思维能力, 因此, 教师为了 
能够完成教学任务, 需要提高教学水平, 以及改变教学 方式方法, 而学生在学习的过程当中, 也应该摆正学习 态度, 并且在学习当中遇到不懂的, 通过自主的和老师 交流沟通, 帮助自己能够更好的学习。同时教师在教学 过程当中, 要注重对大学生的创新思维教学, 激发大学 生的创新思维。

\section{2 具有创造性的教学内容制定}

大学生对于计算机基础课程的掌握能力, 主要体现 在大学生的计算思维。因此教师在进行计算机基础教学 过程当中要提前制定教学大纲, 梳理相关教学知识内 容, 能够拿到在课程当中对大学生进行有效的计算机思 维引导教学。所以教师在进行计算机基础教学过程当 中, 不应该再引用传统的教学模式, 而是需要融入创新 的教学模式方法, 并且在课堂当中要灵活的运用计算机 基础课程知识, 引导大学生的计算思维, 使大学生能够 将计算思维的能力发挥到极致。

\section{6. 结语:}

随着社会高科技的时代的发展, 计算思维能力在你 们的生活日常当中起着越来越重要的作用, 并且逐渐成 为人们在工作上以及学习上的必备技术能力, 而高校教 师如何有效的在进行计算机基础课程教学过程当中引 导学生对于计算思维的学习, 以及大学生如何正确的学 习烹杄及思维, 是我国当代大学生必要的学习内容。

对学生进行计算思维的培养是为了迎合时代的发 展和需求而必须做出的改变, 目前计算机教育教学也正 面临着改革和创新, 而对于非计算机专业的学生而言, 具备计算思维就能够在更大的程度上提高就业优势, 因 此教师应该结合学生的本专业和计算机教学实践, 在此 过程中让学生能够更加了解计算思维, 并培养学生的计 算思维。

\section{REFERENCES}

[1] A study on the cultivation of computational thinking ability in basic computer teaching [J]. Chen Xue. Technology. 2018(13)

[2] A study on the cultivation of computational thinking ability in basic computer science teaching [J]. Wang Le, Wang Fang. Education and teaching forum. 2017(28)

[3] A study on the cultivation of computational thinking ability in basic computer teaching [J ].He Xiaofeng, Li Haiyan, Lu Jiangchun. experimental techniques and management. 2018(09)

[4] A study on the cultivation of computational thinking ability in the teaching of basic computer courses in universities in China [J]. Zhang Jie, Yuan Litian. Wireless Internet technology. 2016(21)

[5] Discussion on Basic Computer Teaching in Universities Based on Computational Thinking [J]. Ren Dan, Ding Xin, Yang Fan. Education and teaching forum. 2018(01)

[6] An Analysis of Basic Computer Teaching in Local Colleges and Universities Based on Computational Thinking $[\mathrm{J}]$. Li Xiaohong, Fan Jiankun, Yang Li. Information and computer (theoretical version).2017(21) 